\title{
Image Edge Extraction by Artificial Intelligence Schemes for UAV Autonomous Navigation
}

\author{
Wanessa da Silva ${ }^{1}$ \\ Nandamudi L. Vijaykumar ${ }^{2}$ \\ Sandra A. Sandri ${ }^{3}$ \\ Haroldo F. de Campos Velho ${ }^{4}$ \\ National Institute for Space Research (INPE), Brazil \\ Zoran Sjanic ${ }^{5}$ \\ University of Linköping, Sweden \\ Elcio H. Shiguemori ${ }^{6}$ \\ Osamu Saotome ${ }^{7}$ \\ Department of Aeronautics Science and Technology (DCTA)
}

\begin{abstract}
We present here the application of an image processing strategy for autonomous navigation in order to estimate the position of an Unmanned Aerial Vehicle (UAV) equipped with Synthetic Aperture Radar (SAR) sensors. Patches of the SAR images are compared to georeferenced satellite optical images, by first applying an image edge detection algorithm on all the images, and the UAV position is determined from the correlation matrix obtained from resulting segmented images. The performance of three edge detectors are compared: the Canny approach, an artificial neural network (radial base function), and a fuzzy system.
\end{abstract}

Key words. SAR images, UAV autonomous navigation, edge detection, image processing.

\section{Introduction}

Unmanned Aerial Vehicle (UAV) is an important emerging technology for several applications. One of the main UAV research topics is the development of autonomous navigation methods, such as those based on image processing. In some of those methods, an image edge detection technique is first applied to both the UAV and georeferenced satellite images; then the UAV position is estimated by comparing the resulting binary images $[1,4]$. This methodology was applied previously to passive sensors for the visible [2] and infrared thermal bands [3], using a MultiLayer Perceptron (MLP), a type of Neural Network (NN).

\footnotetext{
${ }^{1}$ w.nessa.w@gmail.com

${ }^{2}$ vijay.nl@inpe.br

${ }^{3}$ sandri.at.lac.inpe.br@gmail.com

${ }^{4}$ haroldo.camposvelho@inpe.br

${ }^{5}$ zoran.sjanic@liu.se

${ }^{6}$ elcio@ieav.cta.br

${ }^{7}$ osaotome@gmail.com
} 
In [3], Sobel, Canny, and MLP-NN schemes were used for edge extraction, with the UAV position estimated by computing the correlation matrix from segmented images.

In the present study, we also use the edge detection based methodology but focus on Synthetic Aperture Radars (SAR) active sensors, which extend the UAV's operating scenarios for regions with difficult access, such as forest areas, and in case of partial or complete lack of illumination. Moreover, we apply several computational intelligence algorithms for the edge extraction procedure.

Here, SAR data obtained by the UAV is transformed into an image of the worked scenario. The first step is to use an edge extracting technique for the satellite georeferenced and SAR images. The image correlation methods are applied after the process of image edge extraction. The edge extraction procedure is carried out by different techniques: Canny's algorithm, fuzzy system, and neural network - radial base function.

\section{Estimating UAV position by image processing}

The scenario is to determine the UAV position by image processing. The procedure involves: pre-processing, edges detection (image segmentation), and correlation between segmented images. One georeferenced image is from satellite optical sensor and another one is obtained by SAR on board of the UAV.

Image processing of the georeferenced satellite image is described as: a1) a smaller region from the optical image is selected by using coordinates from the INertial Sensor (INS) installed in the UAV, b1) the RGB (red, green, blue) satellite image is mapped into grey scale - some information is lost during the transformation process [5], c1) edge detection: different schemes are employed (Canny, fuzzy system approach, and Radial Base Function - RBF - neural network).

For the UAV image, the steps of processing are expressed as: a2) SAR image is captured by the UAV - a filter is applied on the SAR image to reduce the speckle noise and geometric and radiometric correction - or orthoretification, b2) rotation and scale correction are done, with information from altimeter and compass on board in the UAV, c2) transformation of the UAV image into grey scale, $\mathrm{d} 2$ ) a region is also selected from the image obtained from SAR data, e2) edge detection: the same used on the satellite image.

After the processing on both images mentioned above, a correlation matrix is computed for the segmented images. There are other methods to estimate the UAV position using image processing without correlation, such one employed by Sjanic and Gustafsson [4].

\subsection{Image characteristics}

The reference image is a satellite one obtained by an optical sensor. The employed image was obtained from Google Maps with resolution of $8 \mathrm{~m} /$ pixel, and the image size has $300 \times 600$ pixels.

The SAR (Synthetic Aperture Radar) is a system dealing with emission of electromagnetic (EM) radiation. Different from optical systems, the SAR data is an active sensor to measure the backscatering radiation from its emitted beam. The optical cameras deal 
with additive noise, while the SAR signal is recorded with multiplicative (speckle) noise. A filtering process is executed to reduce the speckle noise, enhacing the signal-noise relation.

We used the SAR aerial image available from the Sandia National Laboratory's (USA: http: //www. sandia.gov). The total size of the SAR image is $810 \times 2000$ pixels. A window from this image is employed with $80 \times 140$ pixels.

\section{Segmentation of SAR Images for Edge Detection}

The segmentation for edge detection deals with the pixel level. In the end, a new image is obtained labeled at 0 or 1 . The values for the pixels are computed considering criteria of similarities, with the goal to mark regions where abrupt changes occur for the grey levels, indicating the boundaries or edges between homegeneous regions with different properties [5].

The edge detection can produce a significant reduction on the amount of data for processing and discards less relevant information, but preserving essential information related to the object shape in the scene. The Canny's algorithm is a procedure already used [1], and details on this scheme can be seen in reference [5].

\subsection{Neural Networks Applied to Edge Extraction}

A key point for using NN as edge extractor lies on the learning phase to identify boundary patterns. For the activation of NN to identify the edge or non-edge pattern, the input data for the $\mathrm{NN}$ is a fixed dimension sliding window traversing all image pixels.

The RBF-NN architecture can be described by an input layer with non-computational units (neurons) receiving the data, where there is only one hidden layer with computational neurons. The activation function is from the radial base function class. We use the Gaussian function. An output layer, with computation neurons (units). There are weighted connections only among the hidden and output neurons.

There are two steps for the learning process to the RBF-NN. Firstly, the self-organizing selection algorithm is applied to determine the centroids. Secondly, the weight connections for the hidden layer is carried out using the back-propagation algorithm [6].

For the learning phase, a set with 26 patterns are employed - see Figure 1. Each pattern is associated with a binary matrix $3 \times 3$, being 24 edge patterns and 2 representing nonedge patterns. For the activation phase, the image to be processed is a $3 \times 3$ mask applied to each pixel. We use this window making the mask traveling pixel by pixel from left to right and from top to bottom, similar to the action for space filtering mask. Each entry of this windown is a NN input. The NN output is an answer to each input data:

- If the NN output is $\left[\frac{1}{0}\right]$, the input pattern represents an edge, and the mask central pixel assumes the value 1 .

- If the NN output is $\left[\frac{0}{1}\right]$, or $\left[\frac{0}{0}\right]$, or $\left[\frac{1}{1}\right]$, the input pattern represents a non-edge, and the mask central pixel assumes the value 0 . 


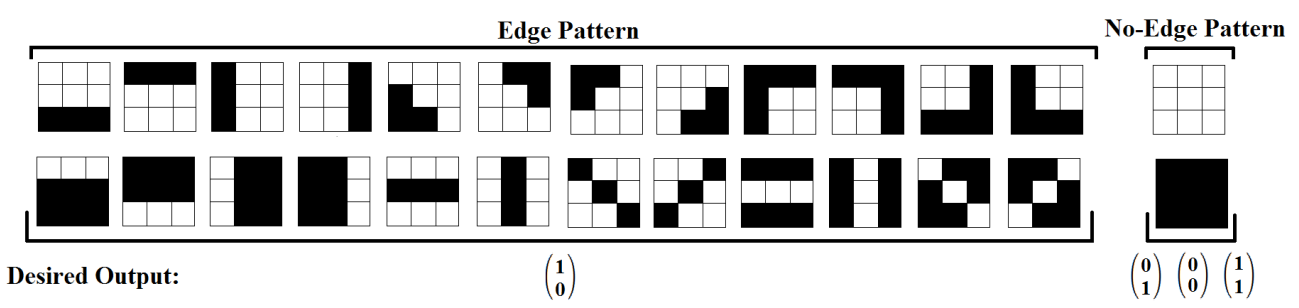

Figure 1: Edge and non-edge patterns employed during the learning phase to the NNs.

\subsection{Fuzzy System Applied to Edge Detection}

Fuzzy systems are based on fuzzy sets theory, where a set of rules are used to perform an inference process. Barkhoda et al. [7] presented a fuzzy edge detector, using the values of gradient (GRAD) for each pixel, and the pixel standard deviation (SD) related to its 8 neighbors. The algorithm is summarized as follows:

1) Sobel's algorithm to compute the gradient (GRAD) for each image pixel;

2) The standard deviation (SD) value calculated for each pixel;

3) GRAD and SD are the input for the fuzzy system - GRAD and SD values are normalized in the interval $[0,100]$. Both linguistic variables GRAD and SD are associated to the same set of fuzzy terms $\{\mathrm{L}, \mathrm{M}, \mathrm{H}\}$. Functions $\mathrm{C}($.$) and \mathrm{S}($.$) denote the core and support$ of fuzzy terms membership functions ${ }^{8}$. Membership functions $\mathrm{L}, \mathrm{M}, \mathrm{H}:[0,100] \rightarrow[0,1]$ are linear by parts with the following cores and supports: $\mathrm{C}(\mathrm{L})=[0,12], \mathrm{S}(\mathrm{L})=[0,37]$, $\mathrm{C}(\mathrm{M})=[37,62], \mathrm{S}(\mathrm{M})=[12,87], \mathrm{C}(\mathrm{H})=[87,100], \mathrm{S}(\mathrm{H})=[62,100]$.

4) The output linguistic variable OUT is associated with three fuzzy terms $\{E L, E M, E H\}$. Membership functions EL,EM,EH: $[0,100] \rightarrow[0,1]$ are also linear by parts with the following cores and supports: $\mathrm{C}(\mathrm{EL})=[0,0.25], \mathrm{S}(\mathrm{EL})=[0,0.5], \mathrm{C}(\mathrm{EM})=[0.5,0.5], \mathrm{S}(\mathrm{EM})=$ $[0.25,0.75], \mathrm{C}(\mathrm{EH})=[0.75,1.00], \mathrm{S}(\mathrm{EH})=[0.5,1.0]$.

5) The fuzzy rules are denoted by triples $(X, Y, Z)$, standing for "If $\mathrm{SD}=\mathrm{X}$ and $\mathrm{GRAD}=\mathrm{Y}$ then OUT=Z" and given by the set $\{(\mathrm{L}, \mathrm{L}, \mathrm{EL}),(\mathrm{L}, \mathrm{M}, \mathrm{EL}),(\mathrm{L}, \mathrm{H}, \mathrm{EM}),(\mathrm{M}, \mathrm{L}, \mathrm{EL}),(\mathrm{M}, \mathrm{M}, \mathrm{EM})$, (M,H,EH), (H,L,EM), (H,M,EH), (H,H,EH)\}.

6) The input entry (GRAD, SD) is mapped on the membership functions and the rules are evaluated.

The relation below is used for the defuzzification process:

$$
P_{\text {final }}=\sum_{a}^{b}\left(E_{\theta} \times C_{j}\right)
$$

where $C_{j}$ is the center of each class in the output membership function. Therefore, $C_{1}=$ $C_{L}=0.25, C_{2}=C_{M}=0.5$, and $C_{3}=C_{H}=0.75 . E_{\theta}$ is obtained by the decision rule showed in Figure 2.

\footnotetext{
${ }^{8}$ Given a fuzzy set $X$, with membership function $X: \Omega \rightarrow[0,1]$, the core and support of $X$ are respectively defined as $C(X) \equiv\{w \in \Omega \mid X(w)=1\}$, and $S(X) \equiv\{w \in \Omega \mid X(w)>0\}$.
} 


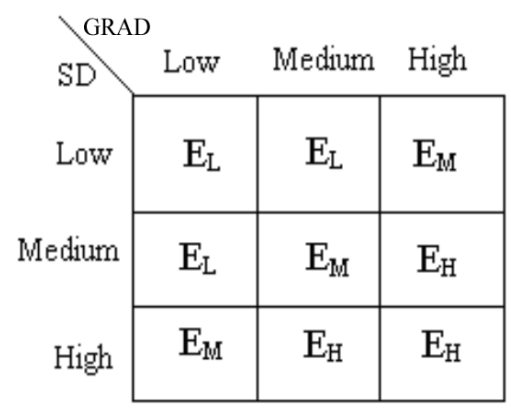

Figure 2: Decision rules of the fuzzy logic algorithm.

\subsection{UAV Position Estimation}

An approach to perform the UAV position estimation by image processing is based on calculating the correlation matrix. The maximum value of the correlation matrix is the UAV position. The correlation matrix is given by [5]:

$$
c(s, t)=\sum_{x} \sum_{y}[f(x, y) \times w(x-s, y-t)]
$$

where $c(s, t)$ is the correlation matrix, $s=0,1, \ldots, M$ and $t=0,1, \ldots, N$, with $M$ and $N$ the dimension of the $f(x, y)$ matrix - the satellite edge image, and the $w(x, y)$ matrix with $J \times K$ dimension, being $J \leq M$ and $K \leq N$, representing the UAV edge image.

The matching between the UAV image $(w)$ and the sector $(f)$ of the satellite image is determined by the convolution matrix. The correlation is computed for each pixel, and the maximum value of the correlation matrix $c(s, t)$ is the UAV position.

\section{Results}

For the analysis of the results obtained with the methodology presented, an experiment is considered using the three different algorithms for edge detection. The experiment consists in estimating the UAV position. The evaluation of the methods is done considering the error of the UAV estimated position. The Euclidean distance is used to compute the error between the true UAV position and the estimated position.

Table 1: Estimation error for UAV positioning with different schemes for edge detection.

\begin{tabular}{l|c}
\hline Edge Detector & Error (meter) \\
\hline Canny & 63.6 \\
Fuzzy system & 40.9 \\
RBF-NN & 34.8 \\
\hline
\end{tabular}

Table 1 presents the error results in meters for the experiment considering different schemes for edge detection. Figure 3 shows result obtained by the correlation operator 

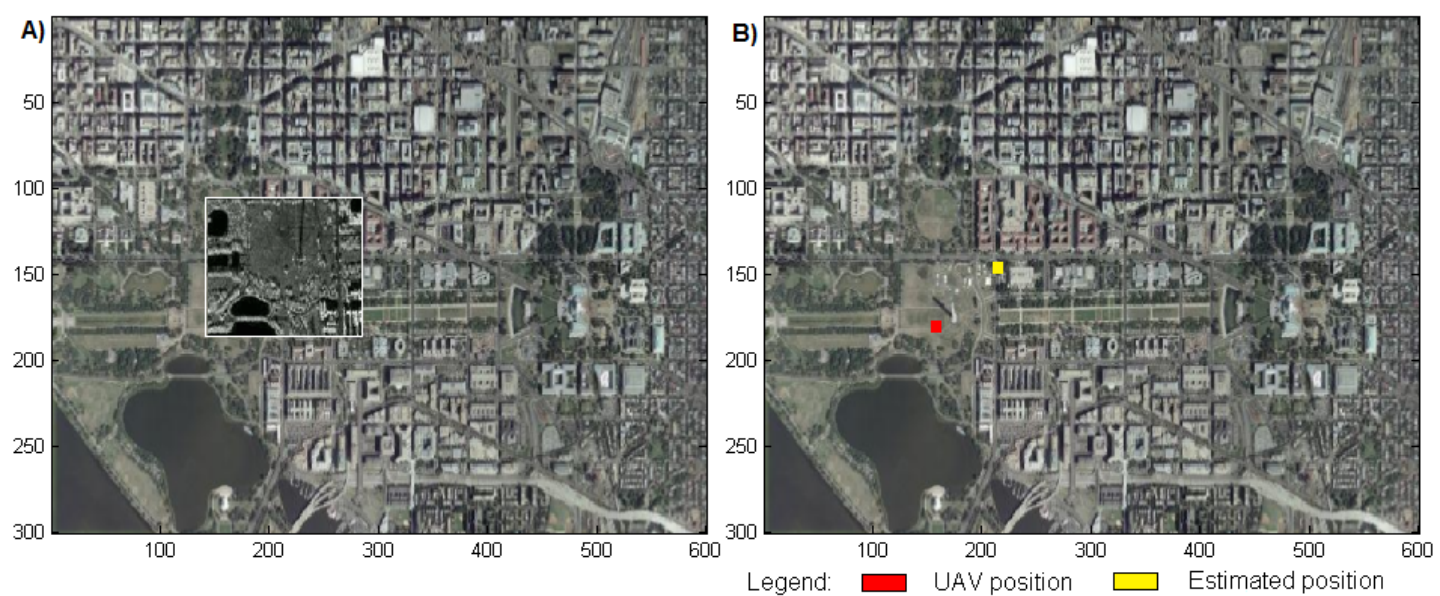

Figure 3: Result for UAV position estimation using RBF-NN.

to estimate the UAV position using the edge images computed by the RBF NN - only the best result is shown. For a better visualization, the results were presented on the original images, but all calculation was carried out with edge images. Figure $3-\mathbf{A}$ shows the correlation between the satellite and UAV images, and Figure $3-\mathbf{B}$ shows the true (red) and estimated UAV (yellow) positions by the correlation coefficient.

\section{Conclusions and Final Remarks}

The study here implements a methodology to estimate the UAV position, employing a pattern recognition approach. The positioning identification is done by correlation between SAR UAV and optical Quickbird satellite segmented images. The goal of our study is to evaluate different methods for edge detection applied to identify UAV positioning. Results were obtained under a search region with size $180 \times 360 \mathrm{~m}^{2}$. The average estimated error was $49.4 \mathrm{~m}$. The algorithm with the best perfomance was the RBF-NN, secondly the fuzzy system, and the worse estimation was obtained with Canny's method. The smallest position estimation error was $27.3 \mathrm{~m}$, obtained by RBF-NN.

To reduce the UAV positioning estimation, the formulation employed by Sjanic and Gustafsson [4] could be employed - instead of correlation matrix. Other different types of NN [9] for edge extraction also deserve to be investigated.

\section{Acknowledgements}

Authors thanks to Capes, Faspep (Proc. 2016/14393-2), and CNPq (Proc. 312924/2017-8) - Brazilian agencies for research support. 


\section{References}

[1] D. Conte and P. Doherty: An integrated uav navigation system based on aerial image matching. IEEE Aerospace Conference. California, USA: IEEE, pages 1-10, 2008.

[2] G. A. M. Goltz, E. H. Shiguemori, and H. F. Campos Velho: Position estimation of uav by image processing with neural networks. 10th Brazilian Congress on Computational Intelligence (G. D. A. Barreto and J. A. F. Costa - Eds.) Fortaleza (CE), Brazil: SBIC, pages 1-8, 2011.

[3] W. Silva, E. H. Shiguemori, N. L. Vijaykumar, and H. F. Campos Velho: Estimation of UAV position with use of thermal infrared images. 9th International Conference on Sensing Technology (ICST). Auckland, New Zealand: IEEE, 2016, pp. 828-833.

[4] Z. Sjanic and F. Gustafsson: Navigation and SAR focusing with map aiding. IEEE Transactions on Aerospace and Electronic Systems, 51: 1652-1663, 2015.

[5] R. C. Gonzalez and R. E. Woods: Digital Image Processing. New Jersey, USA: Prentice Hall, 2002.

[6] S. Haykin, Neural Networks: A Comprehensive Foundation, 2nd Edition New Jersey, USA: Prentice Hall, 1999.

[7] W. Barkhoda, F. Akhlaqian, and O. Shahryari: Fuzzy edge detection based on pixel's gradient and standard deviation values. International Multiconference on Computer Science and Information Technology, Mragowo, Poland, pages 7-10, 2009.

[8] J. R. G. Braga, G. Conte, P. Doherty, H. F. Campos Velho, and E. H. Shiguemori: An image matching system for autonomous UAV navigation based on neural network. XIV International Conference on Control, Automation, Robotics and Vision. Pucket, Thailand: IEEE, pages 1-6, 2016.

[9] E. H. Shiguemori, H. F. Campos Velho, and J. D. S. Silva: Atmospheric temperature retrieval from satellite data: new non-extensive artificial neural network approach. 23rd Annual ACM Symposium on Applied Computing, Fortaleza (CE), Brazil, vol. III, pages 1688-1692, 2008. 